\title{
Advanced photoelectric effect experiment beamline at Elettra: A surface science laboratory coupled with Synchrotron Radiation
}

G. Panaccione, I. Vobornik, J. Fujii, D. Krizmancic, E. Annese, L. Giovanelli, F. Maccherozzi, F. Salvador, A. De Luisa, D. Benedetti, A. Gruden, P. Bertoch, F. Polack, D. Cocco, G. Sostero, B. Diviacco, M. Hochstrasser, U. Maier, D. Pescia, C. H. Back, T. Greber, J. Osterwalder, M. Galaktionov, M. Sancrotti, and G. Rossi

Citation: Review of Scientific Instruments 80, 043105 (2009); doi: 10.1063/1.3119364

View online: https://doi.org/10.1063/1.3119364

View Table of Contents: http://aip.scitation.org/toc/rsi/80/4

Published by the American Institute of Physics

\section{Articles you may be interested in}

A research-based curriculum for teaching the photoelectric effect

American Journal of Physics 77, 87 (2009); 10.1119/1.2978181

A facility for the analysis of the electronic structures of solids and their surfaces by synchrotron radiation photoelectron spectroscopy

Review of Scientific Instruments 88, 013106 (2017); 10.1063/1.4973562

Photoelectric Effect Experiment

American Journal of Physics 29, 706 (1961); 10.1119/1.1937559

Ultraviolet fast-response photoelectric effect in tilted orientation $\mathrm{SrTiO}_{3}$ single crystals Applied Physics Letters 89, 173507 (2006); 10.1063/1.2367658

Efficient spin resolved spectroscopy observation machine at Hiroshima Synchrotron Radiation Center Review of Scientific Instruments 82, 103302 (2011); 10.1063/1.3648102

Design and performance of AERHA, a high acceptance high resolution soft x-ray spectrometer Review of Scientific Instruments 85, 043108 (2014); 10.1063/1.4871362

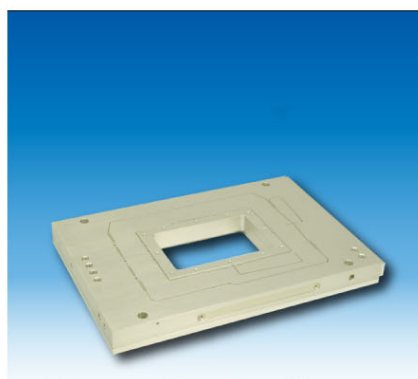

Nanopositioning Systems

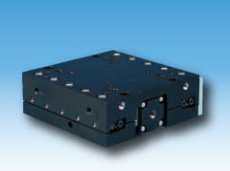

Micropositioning

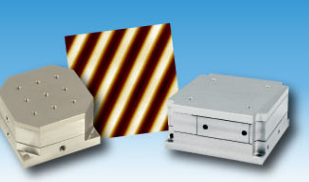

AFM \& SPM

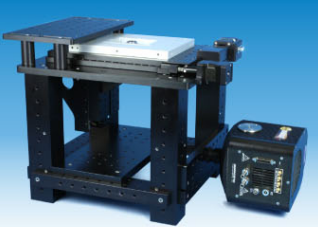

Single molecule imaging 


\title{
Advanced photoelectric effect experiment beamline at Elettra: A surface science laboratory coupled with Synchrotron Radiation
}

\author{
G. Panaccione, ${ }^{1, a)}$ I. Vobornik, ${ }^{1}$ J. Fujii, ${ }^{1}$ D. Krizmancic, ${ }^{1}$ E. Annese,${ }^{1}$ L. Giovanelli, ${ }^{1, b)}$ \\ F. Maccherozzi, ${ }^{1}{ }^{1, c)}$ F. Salvador, ${ }^{1}$ A. De Luisa, ${ }^{1}$ D. Benedetti, ${ }^{1}$ A. Gruden, ${ }^{1}$ P. Bertoch, ${ }^{1}$ \\ F. Polack, ${ }^{2}$ D. Cocco, ${ }^{3}$ G. Sostero, ${ }^{3}$ B. Diviacco, ${ }^{3}$ M. Hochstrasser, ${ }^{4}$ U. Maier, ${ }^{4}$ \\ D. Pescia, ${ }^{4}$ C. H. Back, ${ }^{5}$ T. Greber, ${ }^{6}$ J. Osterwalder, ${ }^{6}$ M. Galaktionov, ${ }^{7}$ M. Sancrotti, ${ }^{8}$ and \\ G. Rossi ${ }^{1, d}$ \\ ${ }^{1}$ TASC Laboratory, INFM-CNR, S.S. 14-Km 163.5 in AREA Science Park, I-34012 Basovizza (Trieste), Italy \\ ${ }^{2}$ Synchrotron SOLEIL, B.P. 48, 91192 Gif-sur-Yvette, France \\ ${ }^{3}$ Sincrotrone Trieste S.C.p.A., S.S. 14 Km 163.5, Area Science Park, 34012 Trieste, Italy \\ ${ }^{4}$ Laboratorium für Festkörperphysik, ETH Hönggerberg, CH-8093 Zürich, Switzerland \\ ${ }^{5}$ Department of Physics, Universität Regensburg, 93040 Regensburg, Germany \\ ${ }^{6}$ Physik-Institut, Universität Zürich, Winterthurerstrasse 190, CH-8057 Zürich, Switzerland \\ ${ }^{7}$ Ioffe Physik-Technical Institute, Polytechnicheskaya 26, St. Petersburg 194021, Russia \\ ${ }^{8}$ Dipartimento di Matematica e Fisica and INFM, Università Cattolica del Sacro Cuore, Via dei Musei 41, \\ I-25121 Brescia, Italy
}

(Received 14 January 2009; accepted 25 March 2009; published online 30 April 2009)

\begin{abstract}
We report the main characteristics of the advanced photoelectric effect experiments beamline, operational at Elettra storage ring, featuring a fully independent double branch scheme obtained by the use of chicane undulators and able to keep polarization control in both linear and circular mode. The paper describes the novel technical solutions adopted, namely, (a) the design of a quasiperiodic undulator resulting in optimized suppression of higher harmonics over a large photon energy range $(10-100 \mathrm{eV}),(\mathrm{b})$ the thermal stability of optics under high heat load via cryocoolers, and (c) the end station interconnected setup allowing full access to off-beam and on-beam facilities and, at the same time, the integration of users' specialized sample growth chambers or modules. () 2009 American Institute of Physics. [DOI: 10.1063/1.3119364]
\end{abstract}

\section{INTRODUCTION}

The past 2 decades witnessed a tremendous technological development aiming at high intensity/high resolution synchrotron radiation (SR) beamlines dedicated to solid state physics. In the particular field of materials and surface science - the forefathers of nanotechnology - an increasing number of third generation SR beamlines operating in the vuv and soft $\mathrm{x}$-ray region (typically 10-1500 eV) have reached the meV range of energy resolution, with $>10^{11}$ photon flux on the sample, thus enabling a vast-and newfield of spectroscopic research. As an added value, the use of variable polarization (linear, circular, and elliptical) and small focal spot by means of new generation insertion devices represents one of the fastest growing fields for electron spectroscopies with SR. The almost complete control achieved over the abovementioned parameters of a beamline points to a new-and twofold - challenge for next generation experiments, i.e., (i) to balance the focused performances of the beamline with the needed flexibility of an experimental

\footnotetext{
${ }^{a)}$ Electronic mail: panaccioneg@elettra.trieste.it.

${ }^{b)}$ Present address: L2MP, Campus de Saint Jérôme, F-13397 Marseille Cedex 20, France and CRMCN-CNRS, Campus de Luminy, Case 913, F-13288 Marseille Cedex 9, France.

c) Present address: Soleil Synchrotron, L'Orme des Merisiers, Saint Aubin 91192, France.

d) Also at Dipartimento di Fisica, Università di Modena e Reggio Emilia, via Campi 213/A, I-41100 Modena, Italy.
}

end station (i.e., share beamtime efficiently) and (ii) to achieve a high level of control in the preparation and characterization of the samples in order to fully exploit the available spectroscopic tools (i.e., use beamtime efficiently).

In this paper we review the performances and the innovative technical solutions of the advanced photoelectric effect (APE) experiments beamline CNR-INFM, a fully operational twin-undulator beamline, located at the Elettra storage

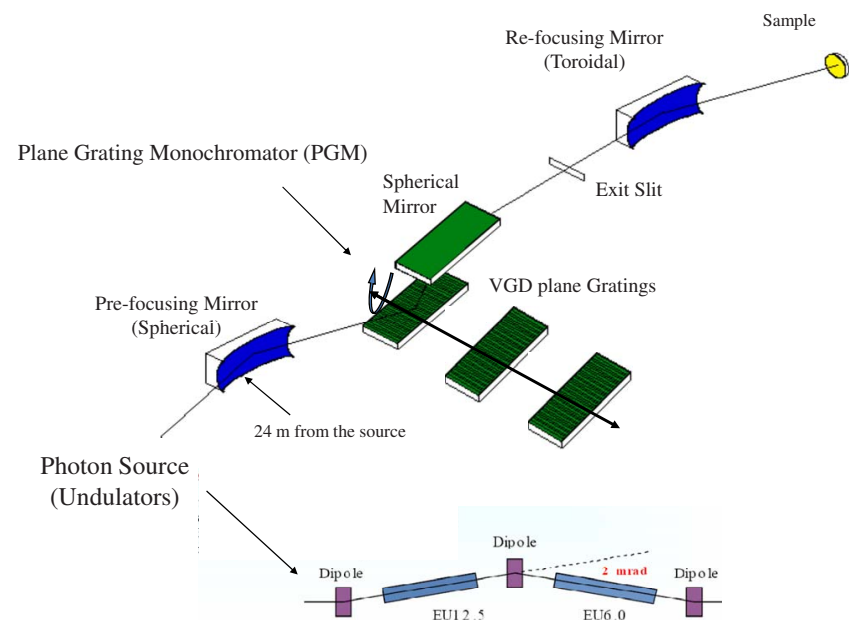

FIG. 1. (Color online) Schematic layout of the optics at beamline APE. Bottom panel: the chicanelike steering of the electron beam for the APE undulators EU12.5 (LE) and EU6.0 (HE). 
a) Top view

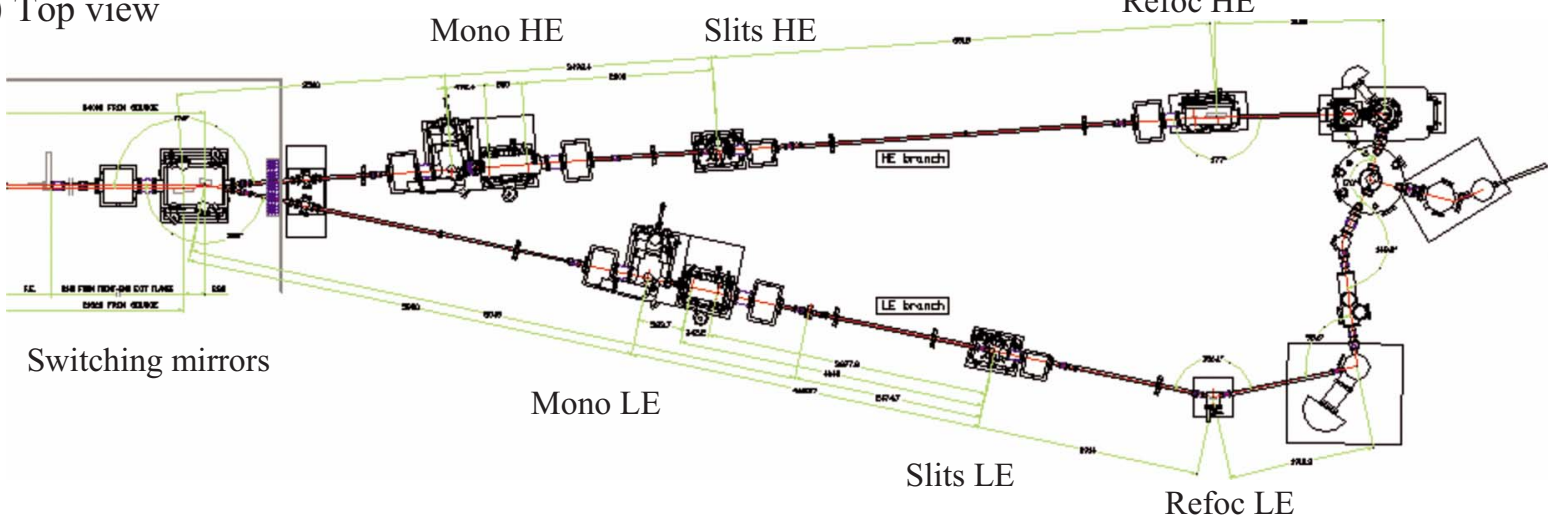

b) Side view

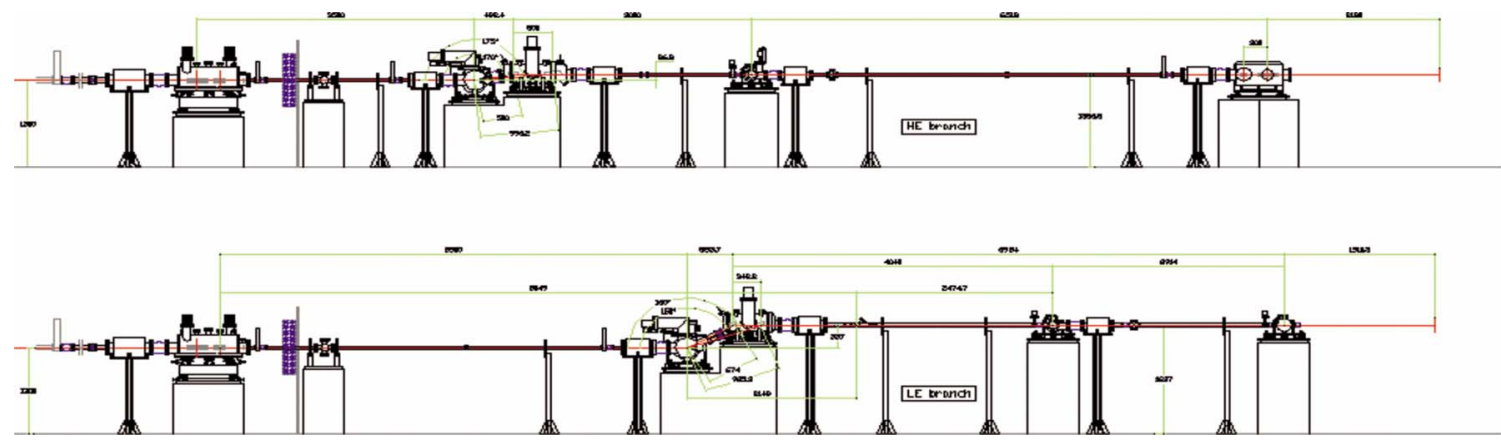

FIG. 2. (Color online) Schematic mechanical layout of the two-branch beamline APE (LE and HE). In both panels (top view and side view), positions of the optical elements are indicated. In panel (a) on the right: the end stations interconnected by several UHV transfer chambers.

ring. The beamline, originated by a Swiss-French-GermanItalian joint collaboration, has been designed with the specific target of matching the characteristics of a surface science laboratory with a fully optimized SR source, resulting in a facility for advanced experiments on solid surfaces and nanostructured matter. The APE beamline uses photons with chosen polarization emitted simultaneously by two noncollinear insertion devices and delivered in two distinct branches able to work independently. The branch dedicated to high resolution photoelectron spectroscopy uses low energy (LE) photons $(h \nu=10-100 \mathrm{eV})$ (APE-LE). The second branch exploits photons in the range of $h \nu=140-1500 \mathrm{eV}$ (APE-HE, HE=high energy) and is mainly devoted to the study of low dimensional magnetic systems.

The paper is organized as follows. In Sec. II we present the novel design features of the beamline, namely, (i) the zig-zag insertion devices arrangement, (ii) the quasiperiodicity of the LE undulator, and (iii) the He-gas cooling system of the first optical elements. The optical layout and the per- formances of the LE and HE branches are discussed in Sec. III. The experimental setup of the end station is presented in Sec. IV. Section V is dedicated to selected results of test experiments.

\section{DESIGN FEATURES}

The sketch and technical layout of the beamline are given in Figs. 1-3. Two variable-polarization undulators are arranged in zig-zag configuration on section number 9 of Elettra storage ring, generating two independent photon beams at a $2 \mathrm{mrad}$ angle in the horizontal plane. One insertion device is quasiperiodic and emits in the far-vuv energy range $(10-100 \mathrm{eV})$; the second insertion emits in the soft $\mathrm{x}$-ray range $(100-2000 \mathrm{eV})$. Each of the two photon beams reaches a first spherical mirror that deviates it into their respective LE or HE branch. Both branches do not use entrance slits. The monochromator section is based on twin plane grating-spherical mirror assemblies. Each monochro-

TABLE I. Optical parameters (LE branch). Angle $\alpha$ is defined as grazing incidence angle. All the LE-branch optics are not coated.

\begin{tabular}{|c|c|c|c|c|}
\hline Nomenclature & $\begin{array}{c}\text { Type } \\
\text { (material) }\end{array}$ & $R, r$, inc. angle $\alpha$ & $\begin{array}{l}\text { Dimensions } \\
\text { (useful area) }\end{array}$ & $\begin{array}{c}\text { Measured slope errors } \\
\text { and surface roughness } \\
(\mathrm{rms})\end{array}$ \\
\hline Lm1 prefocusing mirror & Spherical (Si) & $R=115.4 \mathrm{~m}, \alpha=6^{\circ}$ & $160 \times 17 \mathrm{~mm}^{2}$ & $2.06 \mu \mathrm{rad}, 0.3 \mathrm{~nm}$ \\
\hline Lm2a monochromator mirror & Spherical (Si) & $R=27.4 \mathrm{~m}$ (spec. of 27.38), $\alpha=15^{\circ}$ & $100 \times 20 \mathrm{~mm}^{2}$ & $2.8 \mu \mathrm{rad}, 0.25 \mathrm{~nm}$ \\
\hline Lm2b monochromator mirror & Spherical (Si) & $\begin{array}{c}R=38.38 \mathrm{~m}(\text { spec. of } 38.4), \alpha=10^{\circ} \\
R_{\tan g}=13.38 \mathrm{~m}(\mathrm{spec} . \text { of } 13.47)\end{array}$ & $150 \times 20 \mathrm{~mm}^{2}$ & $3.6 \mu \mathrm{rad}, 0.2 \mathrm{~nm}$ \\
\hline Lm3 refocusing mirror & Toroidal (Si) & $R_{\mathrm{sag}}=0.42 \mathrm{~m}$ (spec. of 0.47$), \alpha=12^{\circ}$ & $70 \times 25 \mathrm{~mm}^{2}$ & $3.5 \mu \mathrm{rad}, 0.4 \mathrm{~nm}$ \\
\hline
\end{tabular}


TABLE II. Optical parameters (HE branch). Angle $\alpha$ is defined as grazing incidence angle. All the HE branch optics are platinum coated.

\begin{tabular}{|c|c|c|c|c|}
\hline Nomenclature & $\begin{array}{c}\text { Type } \\
\text { (material) }\end{array}$ & $R, r$, inc. angle $\alpha$ & $\begin{array}{c}\text { Dimensions } \\
\text { (useful area) } \\
\left(\mathrm{mm}^{2}\right)\end{array}$ & $\begin{array}{c}\text { Measured slope errors } \\
\text { and surface roughness } \\
(\mathrm{rms})\end{array}$ \\
\hline Hm1 prefocusing mirror & Spherical (Si) & $R=302.1 \mathrm{~m}, \alpha=1.5^{\circ}$ & $250 \times 10$ & $1.6 \mu \mathrm{rad}, 0.25 \mathrm{~nm}$ \\
\hline Hm2a monochromator mirror & Spherical (Si) & $R=63.72 \mathrm{~m}$ (spec. of 61.65), $\alpha=2.5^{\circ}$ & $120 \times 20$ & $0.88 \mu \mathrm{rad}, 0.19 \mathrm{~nm}$ \\
\hline Hm2b monochromator mirror & Spherical (Si) & $R=105.16 \mathrm{~m}$ (spec. of 104.6$), \alpha=2.5^{\circ}$ & $180 \times 20$ & $0.74 \mu \mathrm{rad}, 0.19 \mathrm{~nm}$ \\
\hline Hm3 refocusing mirror & Toroidal (Si) & $\begin{array}{c}R_{\tan g}=226.6 \mathrm{~m}, R_{\text {sag }}=120 \mathrm{~mm}, \alpha=1.5^{\circ} \\
R_{\tan g}=121.5 \mathrm{~m}(\text { spec. of } 12.65)\end{array}$ & $250 \times 15$ & $2.2 \mu \mathrm{rad}, 0.3 \mathrm{~nm}$ \\
\hline Hm4 refocusing mirror & Toroidal $(\mathrm{Si})$ & $R_{\text {sag }}=83,2 \mathrm{~mm}($ spec. of 83.7$), \alpha=1.5^{\circ}$ & $70 \times 30$ & $2.2 \mu \mathrm{rad}, 0.4 \mathrm{~nm} \mathrm{rms}$ \\
\hline
\end{tabular}

mator consists of two chambers, one for the gratings and one for the spherical refocusing mirrors. Tables I and II summarize the optical elements and their main optical characteristics. After the water cooled exit slits, both the LE and HE branches illuminate the samples in their end stations via refocusing stages. LE branch uses a single toroidal refocusing mirror, whereas the HE branch can select one of two toroidal mirrors for different focusing options. Great care has been devoted to the stiffness of all the optical mounts in order to guarantee a precise and reliable alignment of the optical elements relative to one another. The natural vibration resonances of the grating and mirror assemblies are above $40 \mathrm{~Hz}$ in order to reduce any influence of floor vibration, which is typically $<40 \mathrm{~Hz}$. Moreover, all the vacuum vessels sit on concrete stands. These concrete stands are cemented to the ground floor and topped by an iron base cemented horizontally. A second base plate is attached to the chamber assembly, sliding on the first base plate for rough horizontal positioning (to within $\sim 0.1 \mathrm{~mm}$ ). The end stations are composed of several interconnected UHV chambers, namely, two end stations with electron spectrometers, three loading/transfer chambers with "dock-ports" to receive user's experimental station and/or dedicated UHV system, and a sample preparation chamber with surface science preparation tools, including a room temperature STM. Samples can be carefully prepared/characterized and, without breaking the vacuum, subsequently analyzed with SR in the analysis chamber(s).

\section{A. Chicane undulators and quasiperiodicity}

Figure 3 sketches the layout of the two undulators (EU6.0, period of $60 \mathrm{~mm}$; EU12.5, period of $125 \mathrm{~mm}$ ) placed in the same straight section; simultaneous use of the two sources is made possible by a chicane e-beam steering. Both undulators (of the APPLE-II-type) ${ }^{1}$ feature variable polarization (linear horizontal, linear vertical, and elliptical) by independent remote control of the magnet arrays with an average switching time $<10 \mathrm{sec}$ (see Fig. 3). Tuning of pho- ton energy and polarization is achieved by mechanically changing, respectively, the vertical gap $(g)$ and the longitudinal phase $\left(Z_{s}\right)$ between top and bottom magnetic arrays. When $Z_{s}=0$ the field is the same as in conventional vertical field undulator producing horizontally polarized radiation. When $Z_{s}=$ period $/ 2$, the magnetic field is horizontal, and the polarization is purely vertical. Elliptical polarization is obtained for values between the two limits of $Z_{s}$. Circular polarization is obtained when horizontal and vertical fields have the same strength. To maintain circular polarization at different photon energies, both $g$ and $Z_{s}$ must be changed. The main undulator parameters are listed in Table III. The chicaned beam path through the two undulators yields two slightly diverging beams separated by only a few millimeters at the front end position. Owing to the difficulty of positioning and aligning two independent pinholes in such a small size, we opted for a single shielding rectangular mask. Finite element analysis calculations have been performed in order to verify that the temperature distribution on the walls of the vacuum chambers remains within acceptable limits in any possible situation. Figure 4 shows the simulated results applied to the photon beam shutter.

The wide vertical power distribution associated with the elliptical fields produces significant heat load on the vacuum chamber and other components, and special procedures must be adopted to minimize the risk of damage. As presented in Fig. 5, linear vertical and circular polarization modes generate to an increased heat load on vacuum chamber, whereas the distribution of horizontal linearly polarized power is confined to the first optics. Two additional sets of movable masks have been inserted between the front end section and first mirror chamber. Such extramask assemblies greatly help in reducing the influence of different heat loading on the walls as a function of the light polarization. Moreover, the presence of additional masks reduces spurious bending magnet radiation along the branches.

One of the two undulators, EU12.5, has been designed

TABLE III. Parameters of LE (12.5) and HE (6.0) undulators. $N$ is the number of periods, $B_{\max }$ is the peak on-axis magnetic field, and $\varepsilon_{1}$ the energy of the fundamental spectral harmonic at the minimum operational gap.

\begin{tabular}{|c|c|c|c|c|c|c|c|c|c|c|c|}
\hline & J on th & & Horizontal & polarization & & Circular & polarization & & Vertical & polarization & \\
\hline$(\mathrm{cm})$ & $(\mathrm{m})$ & $N$ & $B_{0}$ & $\varepsilon_{1}$ & $K_{\max }$ & $B_{0}$ & $\varepsilon_{1}$ & $K_{\max }$ & $B_{0}$ & $\varepsilon_{1}$ & $K_{\max }$ \\
\hline 6.0 & 2.160 & 36 & 0.78 & 59 & 2.8 & 0.42 & 94 & 2.3 & 0.51 & 123 & 4.2 \\
\hline 12.5 & 12.5 & 17 & 0.77 & 8 & 6.9 & 0.48 & 10 & 5.4 & 0.59 & 13 & 8.8 \\
\hline
\end{tabular}


with a nonperiodic array of permanent magnets. We have developed an alternative solution in which the field amplitude (rather than the period) is modulated along the length of the device. ${ }^{2-6}$ This is achieved by selectively removing a few horizontally magnetized blocks, which can be done without any modification to the standard magnet holders. This configuration has been obtained with the specific aim of suppressing high order contribution in the low photon energy regime $(10-80 \mathrm{eV})$ (see section results). A sketch of the quasiperiodic arrangement of permanent magnets in EU12.5 is presented in Fig. 6 together with the figure of merit (flux $\times$ polarization) as a function of photon energy for both EU12.5 (quasiperiodic) and EU6.0 (standard APPLE-II), compared to performances of other undulators in operation at Elettra. Graphics in Fig. 6 present the relationship between $K_{x}, K_{y}$ value, photon energy, gap, and phase values of the quasiperiodic arrangement. Figure 7 shows the measured field distribution in the circular polarization mode, clearly showing the four locations of perturbed field due to the missing blocks. The corresponding computed trajectory is oscillating piecewise along two different axes in a very similar fashion as in the standard variable-spacing quasiperiodic device. A computed spectrum is also presented in Fig. 7 for a realistic case where the relatively large beamline angular acceptance is included. As clearly revealed by the spectra, the intensity distribution presents a fundamental harmonic (i.e., $10 \mathrm{eV}$ in lower graph for circular polarized light), but higher order replicas at integer multiples are absent. In linear mode one observes strong intensities (even more intense than the fundamental) at nonperiodic multiples of the fundamental harmonic, superimposed on a slowly increasing background. Paying the price of a $20 \%$ reduction in flux, a combination of such quasiperiodicity in the undulator and a conventional crystal/grating monochromator provides high spectral purity in the monochromatic photon beam because the noninteger (irrational) quasiharmonics are not diffracted in the same direction by a monochromator. Moreover, no performance penalty has been observed in circular polarization mode. Both in circular and linear modes one observes strong intensities (even more intense than the fundamental one) at nonperiodic multiples of the fundamental harmonic, superimposed to a slowly increasing background.

\section{B. First Mirror cooling system}

A switching mirror chamber common to the two branches houses two silicon spherical mirrors, LEM1 and HEM1, mounted in horizontally deflecting orientation at approximately $24 \mathrm{~m}$ from the source. As the undulators make an angle of $2 \mathrm{mrad}$ with respect to one another, the $\mathrm{HE}$ and LE beams are traveling in a single UHV tube until the location of LEM1 and HEM1, where they are separated by about $48 \mathrm{~mm}$ (see Fig. 8). After this chamber, the two beams enter their respective branches. Both mirrors are referred to a marble plate, acting as an optical reference, through soft bellows.

The mirrors are subjected to a significant heat load (300 Watts at $2.4 \mathrm{GeV}$ with $200 \mathrm{~mA}$ current in the ring). Simulations of different types of cooling have been carried out, showing that the requested optical quality, namely, less than

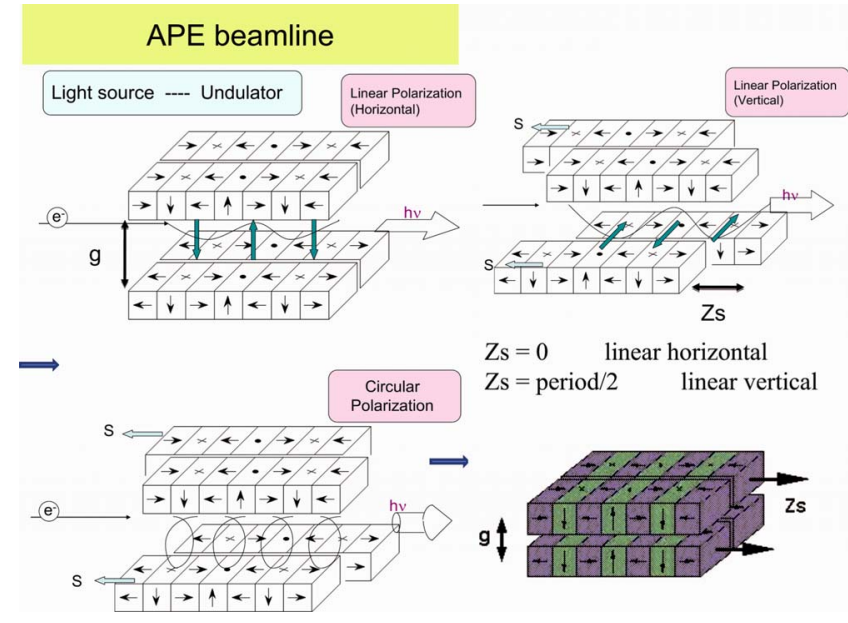

FIG. 3. (Color online) Undulator layout. Schematic of the magnetic structure and polarization control of the APPLE-II-type undulator indicating the mechanical movements of gap and phase ( $g$ and $Z_{s}$, respectively) to obtain linear horizontal, linear vertical, and circular polarized radiations.

$1 \mu \mathrm{rad}$ slope error along the whole active area in both sagittal and meridional directions, cannot be sustained using an external water cooling scheme. To achieve such an optical quality it has been found necessary to cool the silicon down to temperatures where the ratio of thermal expansion to thermal conductivity is much lower than at room temperature. The use of cryocoolers represents a possible approach, knowing that the thermal expansion coefficient of silicon crosses zero at $125 \mathrm{~K} .{ }^{7,8}$ Such an approach has been adopted based on the Gifford-McMahon cooling system, manufactured by Cryomech Inc. of Syracuse, NY, by using a double helium loop for each mirror (coldhead loop and heat exchanger/circulation loop). A specially designed copper block with microcavities is in direct thermal contact with each mirror, leaving the helium circulation outside the UHV, as depicted in Fig. 8. The extracted heat is carried away by the gas and transferred to the coldhead. This design prevents vibrations of the displacer/expander from affecting the pri-
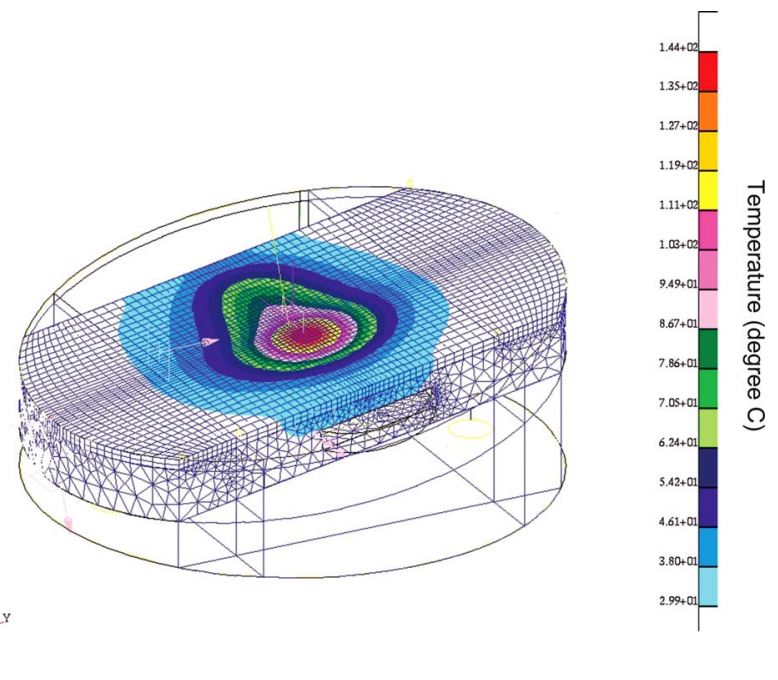

FIG. 4. (Color online) Thermal analysis result for the photon beam shutter with both undulators in horizontal polarization mode, i.e., at maximum power of radiation. Maximum surface temperature is $144^{\circ} \mathrm{C}$. 


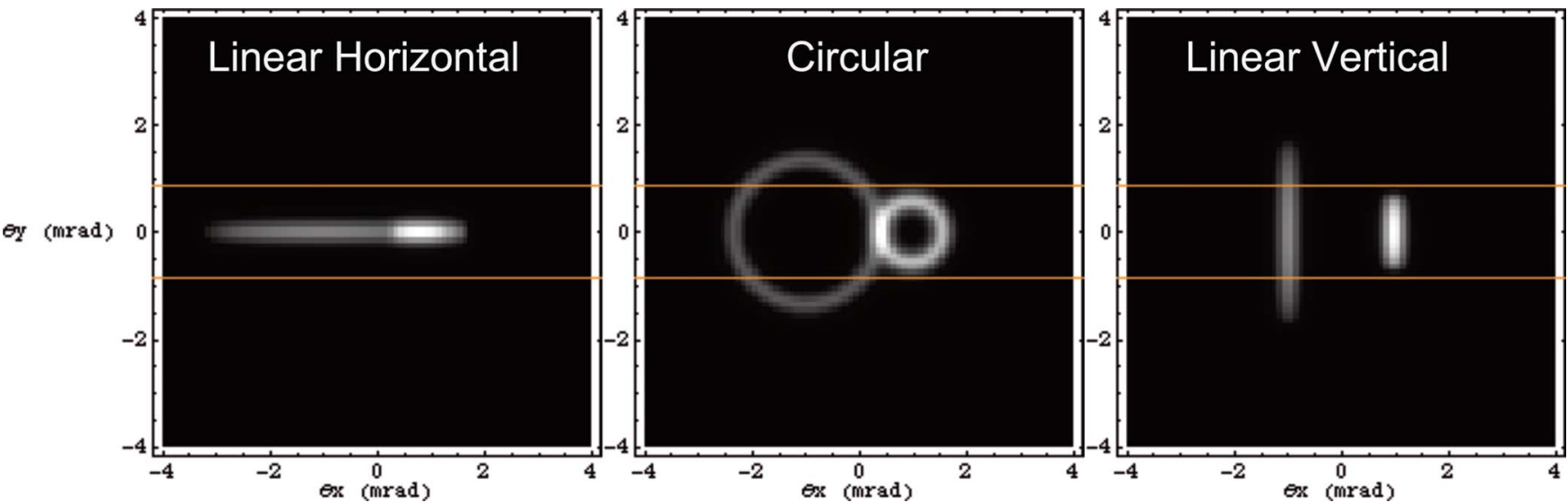

FIG. 5. (Color online) Power density patterns from the two undulators at minimum gap for horizontal $\left(K_{x}=0,0\right)$, circular $\left(K_{x}=5.4 / 2.4\right)$, and vertical $\left(K_{x}=6.9 / 2.9\right)$ polarizations. The yellow lines represent the boundaries corresponding to the part of the radiation cone hitting the vacuum chambers.

mary loop and hence the mechanical stability of the mirrors. With this setup, the temperature reached by the mirrors, as monitored by a thermocouple in close contact with the mirror surface, is below $100 \mathrm{~K}$ under full radiation power, independent of what polarization is used. No influence on both focal spot size and stability of the photon energy has been observed when compressors are in use; this is also due to the decoupling of acoustic vibration made by the concrete block. Mirrors are first connected to the cooling system by copper tubes. After optical alignment, the UHV top plate is positioned, thus leaving the position of the mirrors referenced only to the marble and granite.

\section{BEAMLINE}

The optical layout of both LE and HE branches is presented in Figs. 1 and 2, and the main parameters of the optical surface are summarized in Tables I, II, and IV. Some of the gratings have ruling tracks with different groove depths and densities to optimize harmonic rejection and resolving power in the selected photon energy range. After the installation of the monochromator section, flux and energy resolution tests have been performed using calibrated diodes located after the refocusing section, and an electron multiplier assembly placed after the slits chamber. The degree of circu-
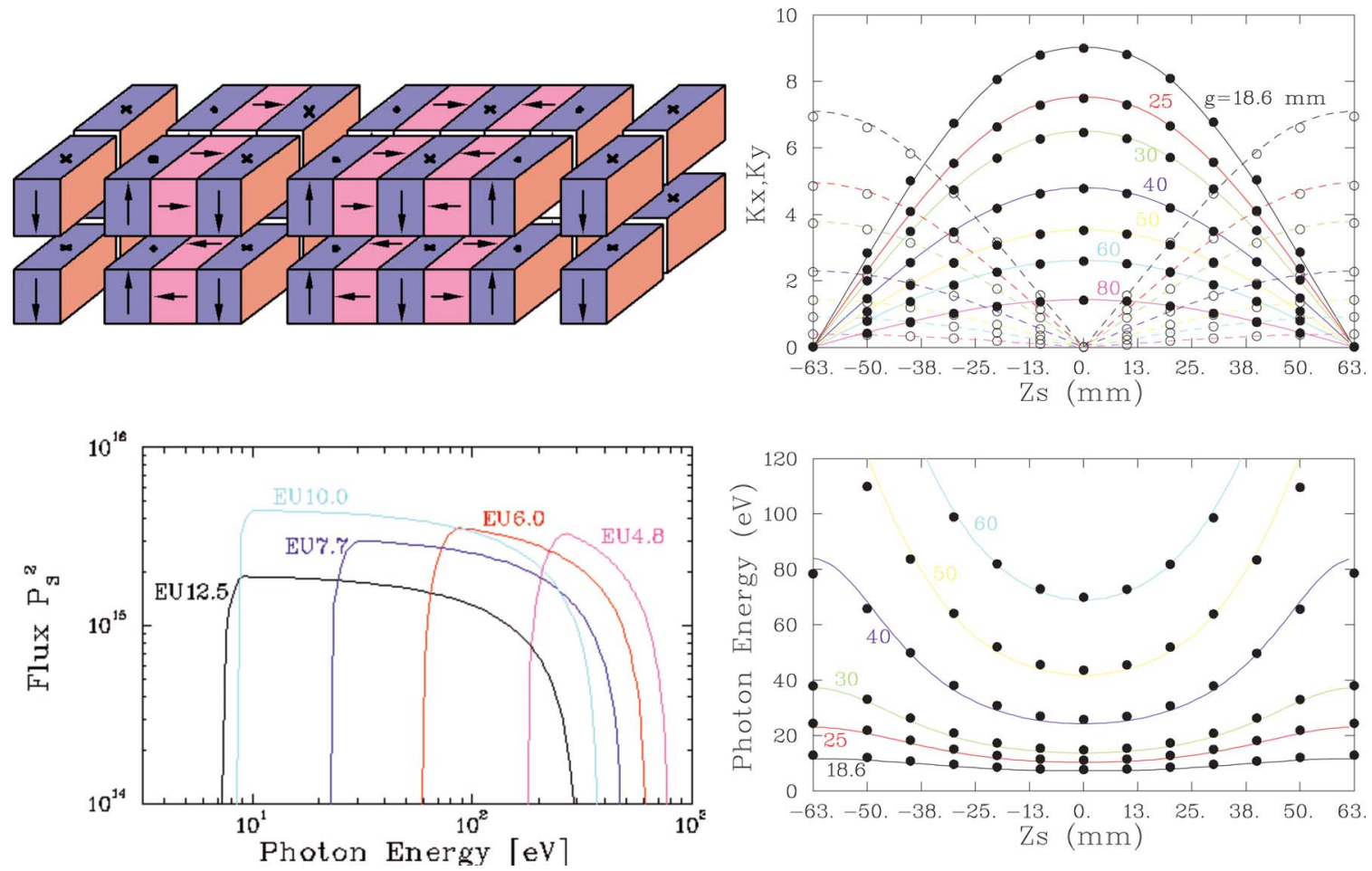

FIG. 6. (Color online) (Top left) Schematic of the magnetic structure of the quasiperiodic LE undulator EU12.5 with low fringe field termination. (Bottom left) Calculated figure of merit and performances of the LE (12.5) and HE (6.0) undulators as a function of photon energy. In linear mode, the figure of merit (polarization rate $\times$ flux) coincides with flux (pol. rate $=1$ ). Right: LE undulator settings. (Top panel) Calculated tables indicating $K_{x}\left(\right.$ black points) and $K_{y}$ (white points) values vs phase shift in millimeter for different gap values. When $K_{x}=K_{y}$ circular mode is obtained. (Bottom panel) Calculated photon energies of fundamental harmonics vs gap and phase shift. 

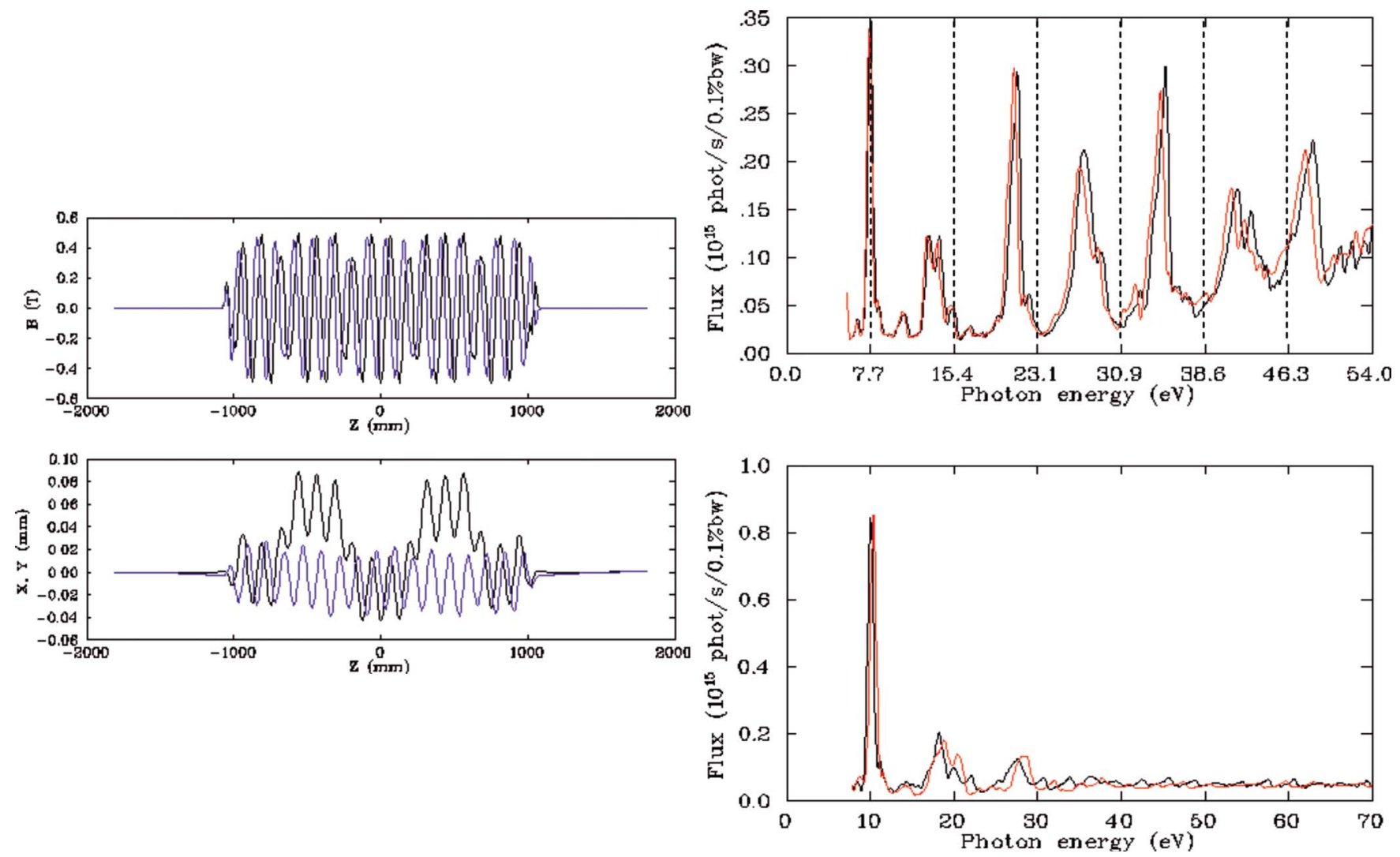

FIG. 7. (Color online) (Top left) Measured field distribution of the quasiperiodic arrangement in circular polarization mode: one notices the four locations of perturbed field due to the missing blocks. (Bottom left) Computed trajectory corresponding to field distribution is along two different axes. Vertical field (horizontal trajectory) shown in black, horizontal field (vertical trajectory) in blue. (Right) Angle integrated spectra of LE (12.5) undulator in linear (top panel) and circular polarization mode (bottom panel) with angular acceptance of $0.7 \times 0.7 \mathrm{mrad}^{2}$. Spectra are computed from ideal (black line) and measured (red line) field.

lar polarization on the sample, for both branches, varies between 0.85 and 0.65 as a function of the used undulator harmonics.

\section{A. LE branch}

Figures 9-11 summarize the main features of the LE branch. Three plane gratings, 700, 1200, and $1600 \mathrm{l} / \mathrm{mm}$, cover the energy ranges of 9-25, 25-40, and 40-100 eV, respectively. The monochromatic light is focused on the vertical plane by a spherical mirror on the exit slit. A toroidal mirror refocuses the light further onto a $50 \times 100 \mu \mathrm{m}^{2}$ spot in the LE end station. All the optic elements of the LE branch are made of pure silicon with no coating. Thus, the intensity has a sharp decrease of around $90 \mathrm{eV}$ photon energy, i.e., close the Si $2 p$ edge. Core level photoemission is still possible up to $120 \mathrm{eV}$, but high resolution/high flux experiments are severely limited above $100 \mathrm{eV}$.

The photon resolution after the exit slit has been determined by measuring absorption spectra of rare gases (He and $\mathrm{Ne})$. We find $E / \Delta E=16000$ at $h \nu \sim 47 \mathrm{eV}$ and $E / \Delta E$ $=13000$ at $h \nu \sim 63 \mathrm{eV}$ for all polarizations. The photon flux at best resolution $(10 \mu \mathrm{m}$ slits $)$ is $>2 \times 10^{10}$ photons/s on the sample, and the overall energy resolution (beamline + spectrometer+temperature) is $6 \mathrm{meV}$, as shown in Fig. 9, for the Fermi edge of a polycrystalline Ag sample. We consistently measure $\sim 8 \mathrm{meV}$ as the light is "switched on" in the range of 15-60 eV of photon energy, while with a slight adjustment of the beamline optics we increase resolution down to $6 \mathrm{meV}$.

As mentioned in the introduction, the LE branch is mainly dedicated to high resolution angular resolved photoemission and Fermi surface mapping. Being the APE beamline design has no entrance slit, any variation in the source position or large angular variation at the monochromator would introduce a shift in the photon energy, thus compromising the reliability of the results. Stability of the beam versus time and versus photon energy range has been then carefully tested. Results are presented in Fig. 10, where the Fermi level of a reference sample measured over a long time interval is contrasted with results obtained with a discharge He-lamp in the same experimental conditions. The stability of the photon energy is such that we find $<0.5 \mathrm{meV}$ shift during roughly one $8 \mathrm{~h}$ shift in beamtime.

Having ascertained the stability and the energy resolution of the LE branch, Fig. 11 displays the spectral shapes of quasiharmonics over the entire region covered by the three gratings. One notices that, as expected, only the fundamental harmonic is intense with no evident higher orders. The importance of a quasiperiodic structure is confirmed by Fig. 12, where the $d$-band and Fermi level (mainly $s$ - $p$ states) of a Ag single crystal sample are displayed, measured at a photon energy of $25 \mathrm{eV}$. In the inset, the Fermi level is located at $20.7 \mathrm{eV}$. The spectrum extends over a larger kinetic energy, 


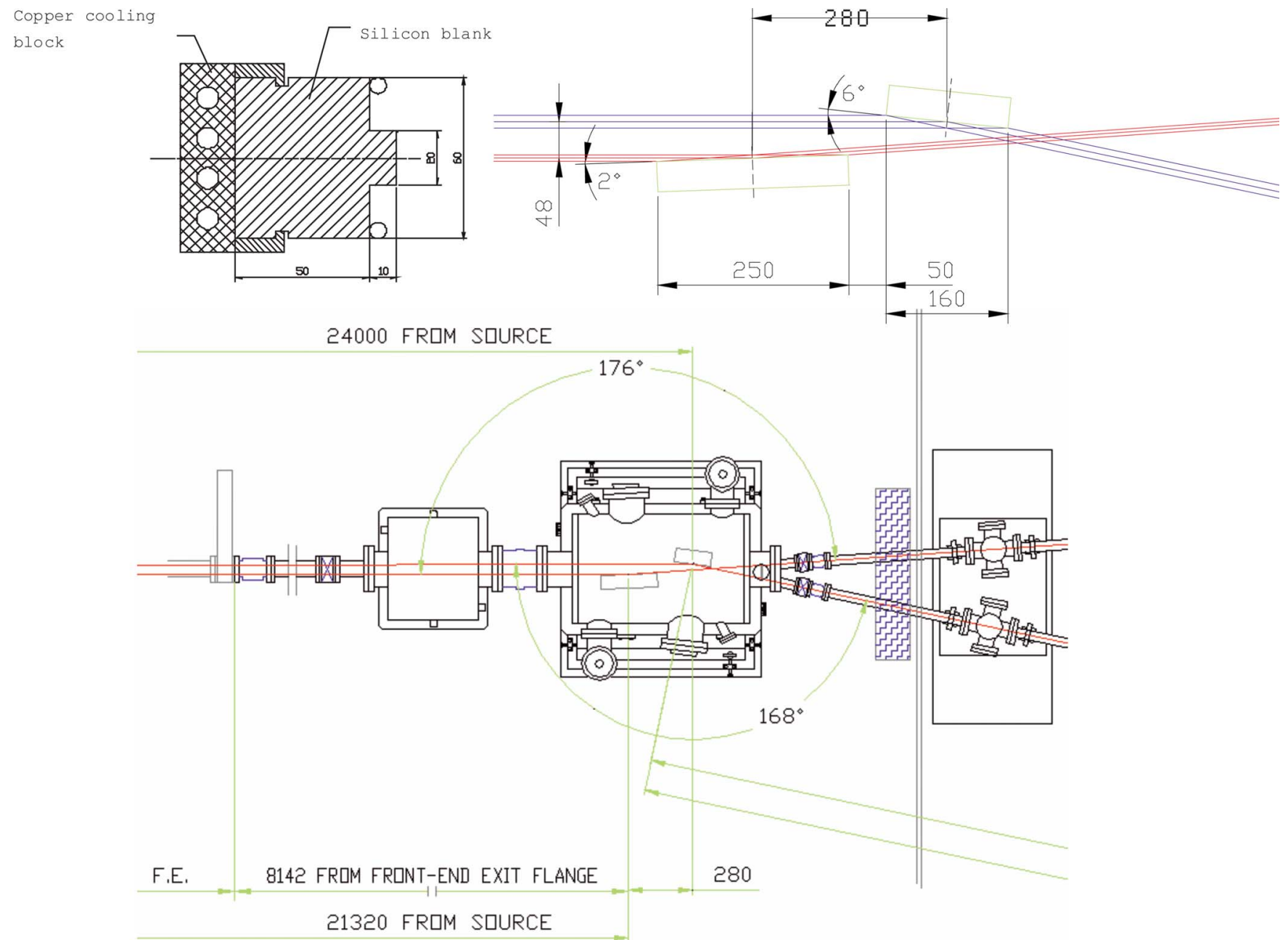

FIG. 8. (Color online) Details of both mounting and cooling system of the first spherical mirrors HEM1 and LEM1. The mirrors are accommodated in the same UHV vessel. The two beams from HE and LE undulators are separated by $48 \mathrm{~mm}$.

in order to detect also the possible contribution of a second order radiation, expected at $50 \mathrm{eV}$. Knowing that at $50 \mathrm{eV}$ photon energy the photoionization cross section of $\mathrm{Ag} 4 d$ is $10^{4}$ times higher with respect to one of the $s$ levels, the

TABLE IV. Grating characteristics. LE gratings are made of pure silicon; HE gratings are platinum coated. Radius of the whole set of gratings is $R$ $>25 \mathrm{~km}$. Slope error and surface roughness are, respectively, $0.5 \mu \mathrm{rad}$ and $0.25 \mathrm{~nm}$ (rms values).

\begin{tabular}{|c|c|c|c|c|}
\hline Nomenclature & $\begin{array}{l}\text { Energy } \\
\text { range }\end{array}$ & $\begin{array}{l}\text { Deviation } \\
\text { angle } \\
\left(^{\circ}\right)\end{array}$ & $\begin{array}{l}\text { Line density } \\
(1 / \mathrm{mm}) \\
\text { and groove } \\
\text { depth } \\
(\AA)\end{array}$ & $\begin{array}{c}\text { Dimensions } \\
\left(\mathrm{mm}^{2}\right)\end{array}$ \\
\hline LE1 first track & $10-20 \mathrm{eV}$ & 150 & 700,900 & $80 \times 10$ \\
\hline LE1 second track & $15-25 \mathrm{eV}$ & 150 & 700,800 & $80 \times 10$ \\
\hline LE2 first track & $20-35 \mathrm{eV}$ & 150 & 1200,300 & $80 \times 10$ \\
\hline LE2 first track & $35-45 \mathrm{eV}$ & 150 & 1200,420 & $80 \times 10$ \\
\hline LE3 single track & $35-80 \mathrm{eV}$ & 160 & 1600,260 & $90 \times 10$ \\
\hline HE1 first track & $180-500 \mathrm{eV}$ & 170 & 1800,70 & $120 \times 10$ \\
\hline HE1 second track & $1000-2000 \mathrm{eV}$ & 175 & 1800,50 & $240 \times 10$ \\
\hline HE2 first track & $150-400 \mathrm{eV}$ & 170 & 1400,130 & $110 \times 10$ \\
\hline HE2 second track & $600-1600 \mathrm{eV}$ & 175 & 1400,60 & $190 \times 10$ \\
\hline HE3 single track & $400-2000 \mathrm{eV}$ & 170 & 900,110 & $190 \times 10$ \\
\hline
\end{tabular}

intense $\mathrm{Ag} 4 d$ band should be better visible with a sizeable second order contribution. The arrows indicate such contribution, revealing that the higher order component is limited to $\approx 1 \%$ of the Photo Emission Spectroscopy intensity of the first quasiharmonic with no detectable third or fourth order contributions.

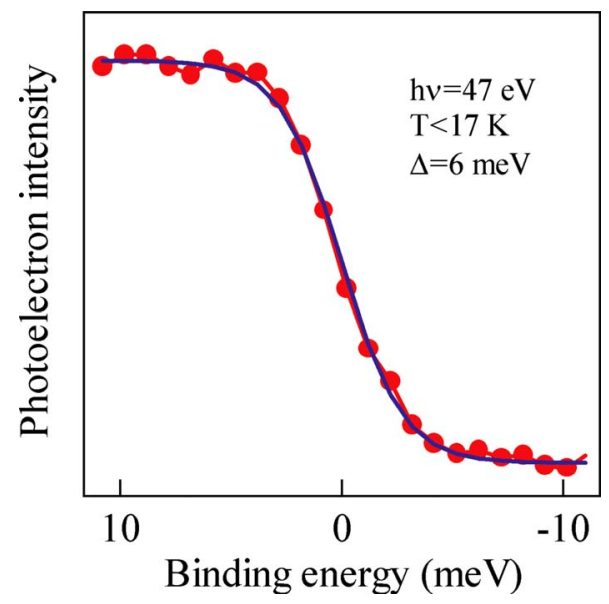

FIG. 9. (Color online) Fermi level from polycrystalline Ag measured at $h \nu=47 \mathrm{eV}$ and $16 \mathrm{~K}$. The red line is the best fit obtained, revealing an overall experimental broadening of $<6 \mathrm{meV}$. 

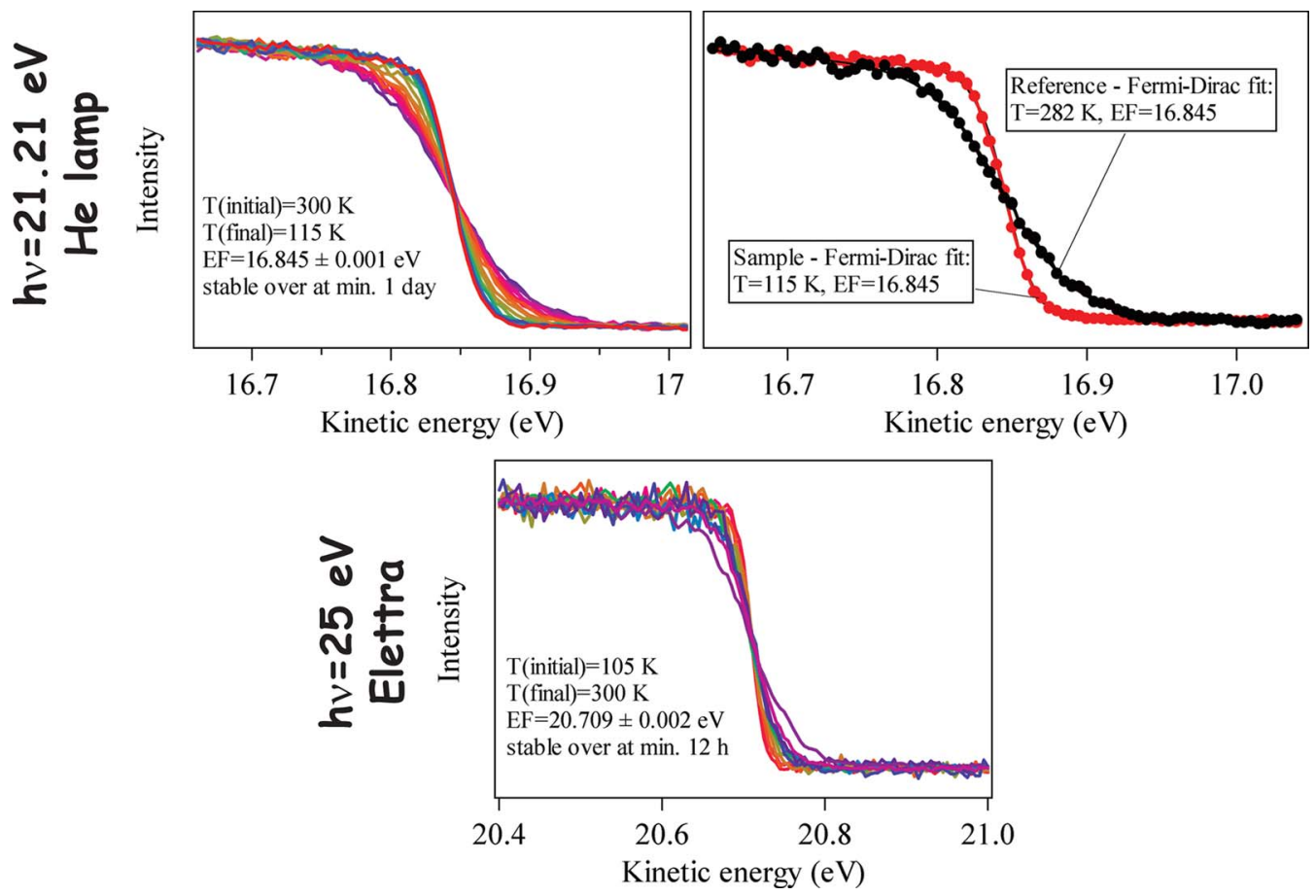

FIG. 10. (Color online) Beam stability test. Series of Fermi level measured from poly-Ag as a function of time and probe. Top: reference vs time measurements obtained via He-discharge lamp $(h \nu=21.2 \mathrm{eV})$, revealing a stability of $1 \mathrm{meV}$ over $24 \mathrm{~h}$. Bottom: same test with SR at $h \nu=25 \mathrm{eV}$. Results indicate a $2 \mathrm{meV}$ stability over $12 \mathrm{~h}$.

\section{B. HE branch}

Similar to LE branch design, the light from the periodic EU6.0 Apple II undulator is horizontally deflected by a spherical mirror onto the plane grating monochromator, which covers the 140-1500 eV energy range (900, 1400, $1800 \mathrm{l} / \mathrm{mm}$, double track with variable groove density). Also in the case of the HE branch, the spherical mirror of the monochromator focuses the light on the exit slit in the vertical plane. The horizontal size of the beam can be adjusted down to $100 \mu \mathrm{m}$ by means of a removable dove tail assembly. Two focusing schemes can be alternatively chosen when operating APE-HE: single reflection focusing by a toroidal mirror that produces a spot size of $75 \times 150 \mu \mathrm{m}^{2}$ or deviation by a second toroidal mirror onto a Fresnel zone plate diffractive optics (under development). Characteristic harmonic scans across the available photon energy range are presented in Fig. 13. The photon resolution after the exit slit has been determined by measuring $\mathrm{N}_{2}$ and $\mathrm{Ne}$ absorption spectra in gas phase. We find $E / \Delta E>8000$ at $h \nu \sim 400 \mathrm{eV}$ and $E / \Delta E \approx 3000$ at $h \nu>900 \mathrm{eV}$ with a photon flux of $>5 \times 10^{10}$ photons/s at best resolution in the range of $200 \mathrm{eV}<h \nu<450 \mathrm{eV}$, decreasing down to 1 $\times 10^{10}$ photons $/ \mathrm{s}$ at $h \nu>1200 \mathrm{eV}$.

\section{END STATIONS}

A $3 \mathrm{D}$ rendering of the APE end stations and preparation laboratory is presented in Fig. 14. The setup allows the integration of users' specialized sample growth chambers or modules, which are connected to the main sample distribution chamber and have full access to the off-beam and onbeam facilities. Substrates or samples can be loaded into the

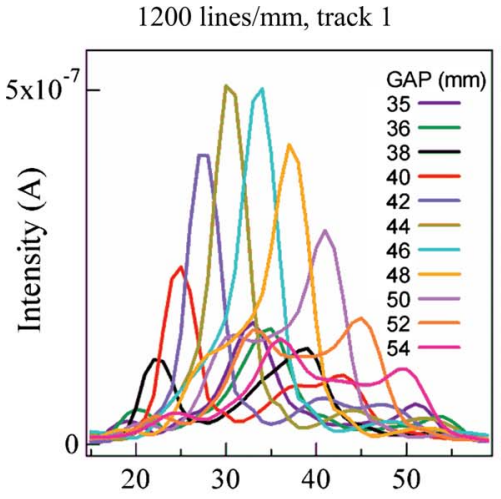

1200 lines $/ \mathrm{mm}$, track 2

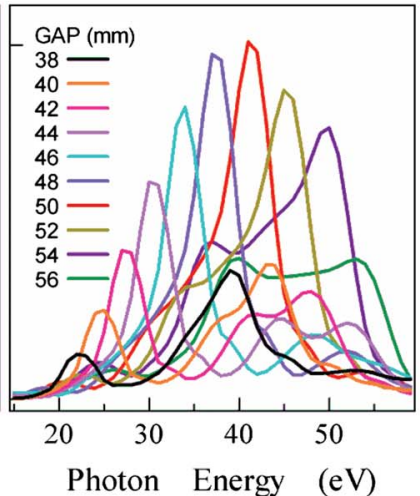

1600 lines $/ \mathrm{mm}$

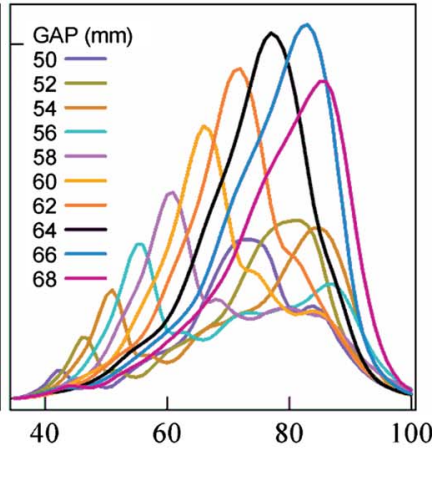

FIG. 11. (Color online) Intensity of LE quasiharmonics as measured by a calibrated diode with $2 \mathrm{GeV}$ and slits of $25 \mu \mathrm{m}$. From left to right: lineshapes are presented as a function of gap value and photon energy for three gratings (1200 1/mm, track 1 and 2, and 1600 1/mm). 


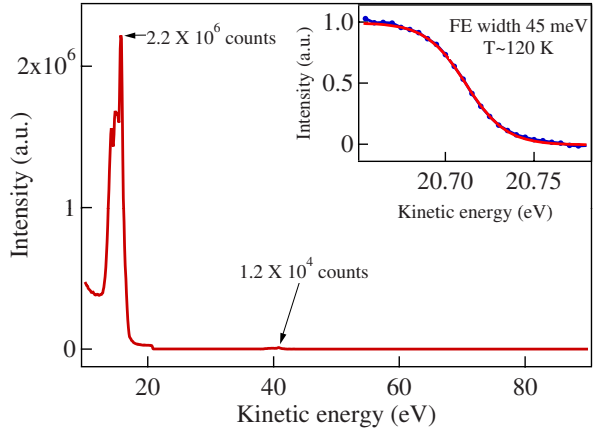

FIG. 12. (Color online) Contribution of higher orders at LE branch. Extended valence band spectra from $\operatorname{Ag}(100)$ at $h \nu=25 \mathrm{eV}$. Counts measured on the maximum of the $\mathrm{Ag} 4 d$ states are indicated, and the inset shows a zoom into the region of the Fermi level around $20.7 \mathrm{eV}$ of kinetic energy. The region spans over the possible second and third order contribution, showing roughly $1 \%$ overall contribution, as indicated by the arrow and number of counts measured at the location of the second order for $\mathrm{Ag} 4 d$.
APE system via two differentially pumped load-locks and then transferred in UHV to and from any of the preparation chambers, to the STM, and/or to both the SR spectrometers.

\section{A. LE end station}

The APE-LE experiments are controlled by software that dialogs with the monochromator actuators and the Elettra beamline control system (undulator gap and phase, i.e., energy and polarization control) with the Scienta SES 2002 analyzer control as well as other ancillary equipment. This end station is also equipped with a He discharge lamp, reverse-view Low energy electron diffraction (LEED), and Ar sputter gun and is compatible with compact evaporation sources. More complex sample treatments can be done in the annex preparation chamber(s). Depending on the type of the experiment, two alternative manipulators are available at
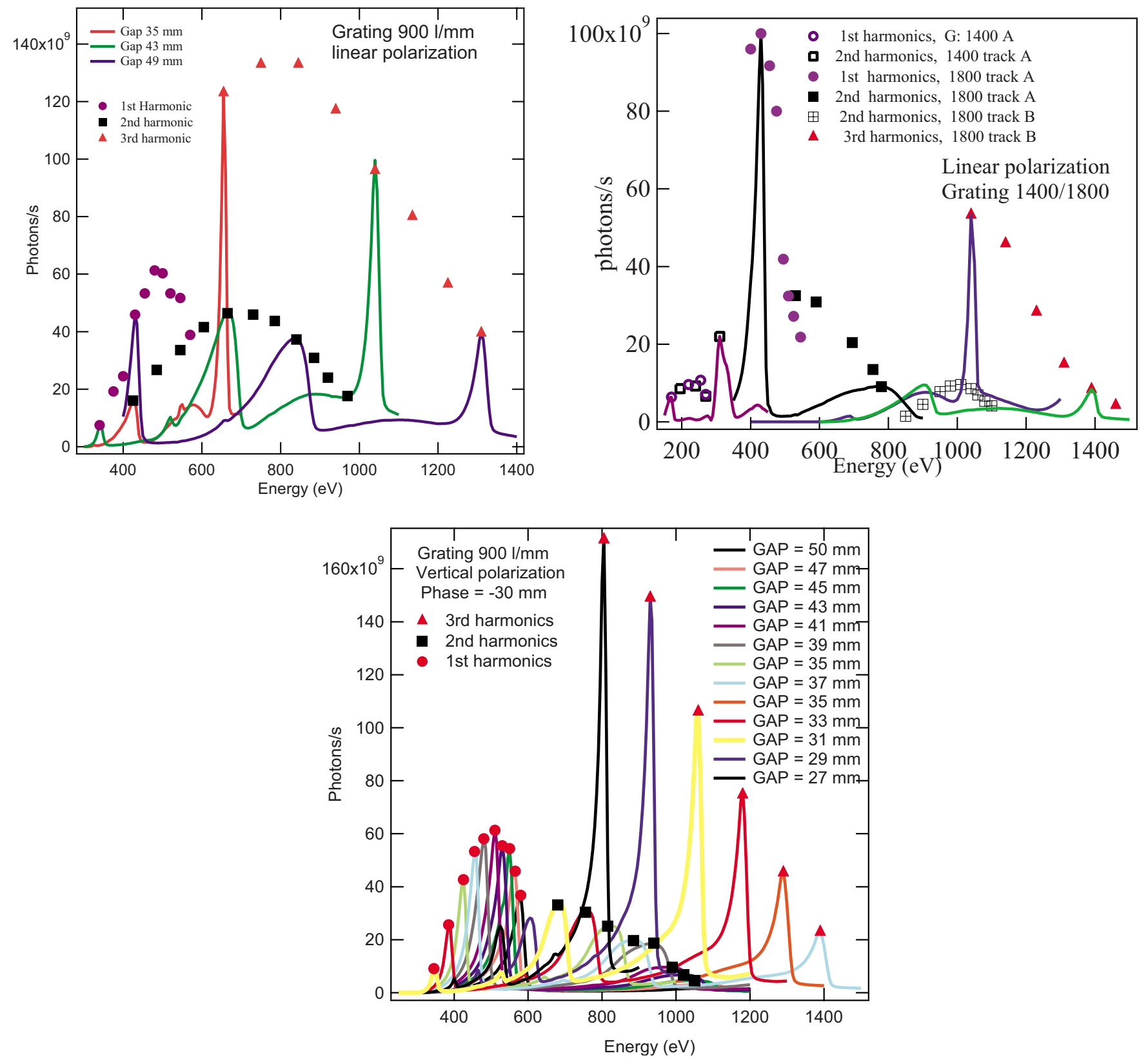

FIG. 13. (Color online) Intensity of HE harmonics as measured by a calibrated diode with $2 \mathrm{GeV}$ and slits of $50 \mu \mathrm{m}$. Grating and polarization are indicated in each panel. Points correspond to measured intensity at fixed gap value with different harmonics (first, second, and third). 


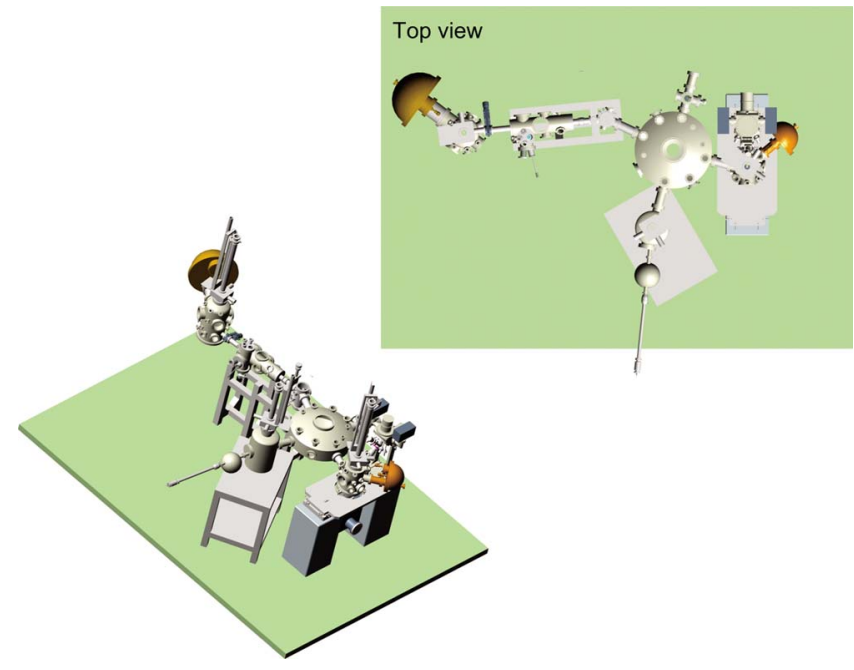

FIG. 14. (Color online) 3D artistic view of the end stations and preparation chambers.

APE-LE: (1) a manually operated one with $x, y, z, \theta$, and $\varphi$ (polar and azimuthal angles) degrees of movement, hosting a home-made cryostat reaching $\sim 15 \mathrm{~K}$ on the sample surface, and (2) a translator containing the automated manipulator (built at the University of Zürich) with the $x, y, z, \theta$, and $\varphi$ degrees of movement. This manipulator is optimized for the automated Fermi surface mapping. The gear-based control of the angular degrees of freedom raises the minimum temperature obtainable on this manipulator to $\sim 150 \mathrm{~K}$.

The two-dimensional detector, such as the one provided with the Scienta SES2002 analyzer, allows for the simultaneous acquisition of the electronic band dispersion versus polar angle (over a $\pm 7^{\circ}$ range in the present case) around the nominal polar angle and along the direction set by the sample azimuth. At given $\theta$ and $\varphi$, the photoelectron intensity measured within one scan at the constant kinetic energy (e.g., Fermi energy) corresponds to the intensity at a circular arc in the reciprocal space. In order to reconstruct the Fermi surface, it is necessary to make subsequent scans for a sufficiently large number of $(\theta, \varphi, h \nu)$ values in order to cover at least one three-dimensional Brillouin zone of the material under investigation. The energy and $k_{\|}$(momentum parallel to the surface) resolution are set by the monochromator and energy analyzer properties, while the $k_{\perp}$ (momentum perpendicular to the surface) resolution depends on the photon energy step chosen for obtaining subsequent data sets.

Our acquisition system coordinates the movement of the manipulator and the subsequent analyzer acquisition in a way that for each photon energy, the desired portion of the reciprocal space is mapped. It organizes the data saving (a typical data file size for a single photon energy is between 20 and 50 Mbytes, depending on the size of the surface Brillouin zone) and the data export in a format convenient for the data analysis using commercially available software. An example of the three-dimensional Fermi surface mapping is shown in Fig. 15, where two perpendicular planar cuts of the Fermi surface of $\mathrm{Cu}(111)$ are displayed.

\section{B. HE end station}

The APE-HE experimental setup makes feasible qualitative and quantitative analyses concerning sample composi-
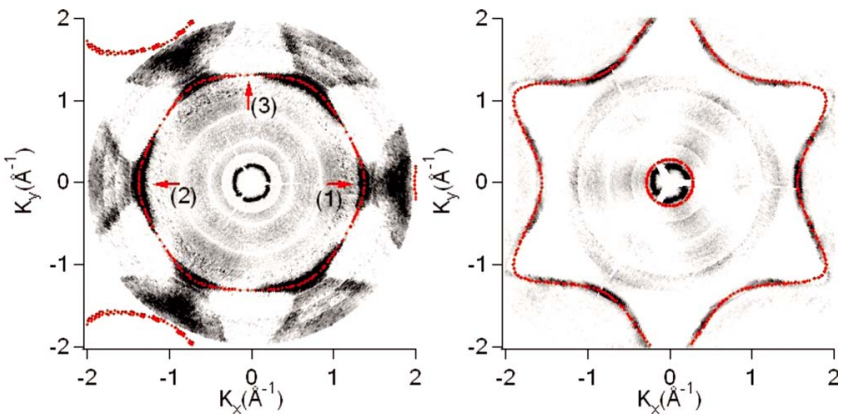

FIG. 15. (Color online) Fermi surface mapping from $\mathrm{Cu}$. Planar cuts through the $\mathrm{Cu}$ Fermi surface in (111) plane (parallel to the sample surface). $k_{z}=3.01 \AA^{-1}$ (left), $k_{z}=4.52 \AA^{-1}$ (right). Red contours: tight binding model calculation.

tion (core level photoemission) and its magnetic properties (x-ray magnetic circular dichroism (XMCD) and spin polarization of ejected photoelectrons). The HE chamber is built for high mechanical stability in order to decouple vibrations during sample positioning and rough scanning $(0.5 \mu \mathrm{m}$ resolution provided by external motors). The APE-HE spectrometer is fully $\mu$-metal lined. Sample preparation is performed in UHV-connected chamber(s). The sample motion has four degrees of freedom: the motorized $x, y, z$, and the manually operated $\theta$ (polar angle). The sample can be cooled down to $25 \mathrm{~K}$ via a liquid-He flow cryostat.

The manipulator is equipped with an UHV compatible electromagnet, providing the magnetic field of up to a maximum of $0.1 \mathrm{~T}$ (pulsed) in a $6 \mathrm{~mm}$ gap, where the surface of the sample is located. The magnetic field can be reversed between the two possible senses along one direction (parallel to the short edge of the sample holder) in the sample surface plane. X-ray magnetic circular and linear dichroisms are performed in both surface sensitive mode (total electron yield in current mode) and bulk sensitive one (fluorescent photon yield). X-ray Absorption Spectroscopy and XMCD maps throughout the surface of the sample can be acquired at fixed photon energy, an effective tool for wedge-shaped and small specimens. The ejected electrons are analyzed in energy by a hemispherical electron energy analyzer equipped with 7 channeltrons (Omicron EA125). The angle between the incident photon beam and the analyzer is fixed and equal to $45^{\circ}$.

A twin Mott scattering setup is available in the chamber for vectorial determination of the spin polarization of secondary electrons, a quantity directly proportional to the remanent long range magnetization of the sample. ${ }^{9,10}$ A sketch of the Mott detectors assembly is presented in Fig. 16. We use conventional $60 \mathrm{kV}$ spherical polarimeter in which both the scattering target (a thin gold foil) and detectors (passivated implanted planar silicon) are held at the same potential. Therefore scattering of the electrons occurs in a field-free environment. The two polarimeters are arranged in a $90^{\circ}$ setup. Knowing that Mott polarimeters can only detect polarization transverse to the direction of electron travel, such a twin assembly allows a full determination of the magnetization vector of the sample. A specially designed electrostatic optics lens and fast electrostatic switch have been coupled to the polarimeters to optimize transmission and intensity of low kinetic energy electrons. ${ }^{11,12}$ Figure 16 reports results 
a) $\mathrm{Fe}(001)$ substrate
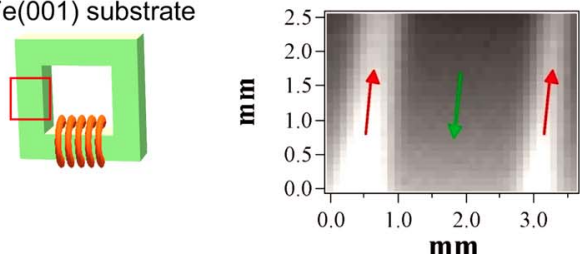

c) Spin resolved secondary electron yield
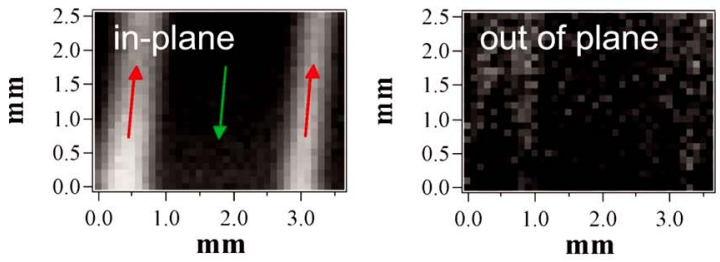

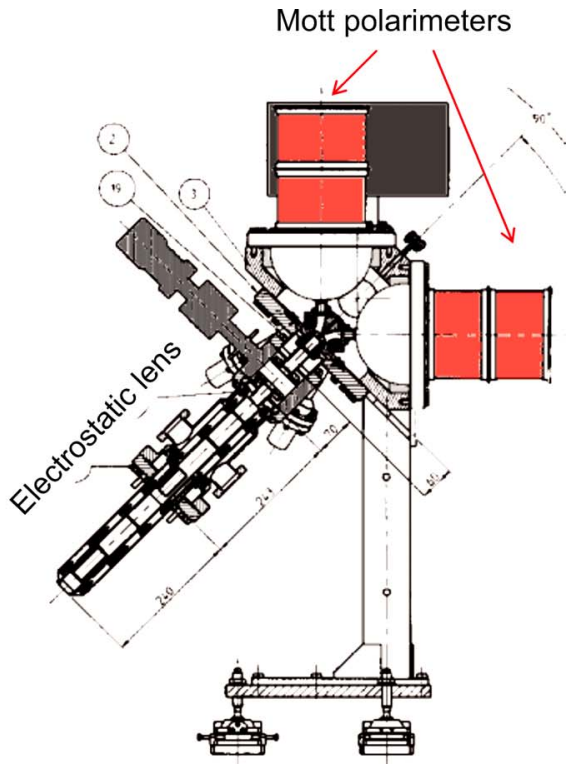

FIG. 16. (Color online) Example of $\mathrm{x}$-ray based spectroscopy performed at APE-HE. (a) sketch of the picture frame Fe(001) sample. Scanned area is indicated by the red square. (b) XMCD as measured in total electron yield at the $L 3$ edge maximum absorption of Fe. White and dark areas correspond to different Fe domains whose orientation along the (001) direction is indicated by green and red arrows. (c) Spin-polarization maps of the same sample area as measured by Mott detector scattering along the $\mathrm{Fe}(001)$ and (010) directions, respectively. Out of plane magnetization is absent, whereas in-plane magnetization well reproduce the XMCD results.

obtained on a $\mathrm{Fe}(100)$ picture frame detected by scanning the sample under the beam, using XMCD and Mott detector, where the in-plane remanent magnetization was oriented along the $\mathrm{Fe}(001)$ direction. The APE-HE experiments are efficiently controlled by an in-house LABVIEW software, which coordinates the Elettra beamline control system (undulator gap and phase, i.e., energy and polarization control) and apparatus (monochromator, Omicron EA125 analyzer, magnetic field, electrometers, position motors, and Mott analyzer). ${ }^{11-14}$

\section{Kerr-STM preparation chamber}

The sample preparation chamber is equipped with heating stages (up to $1500 \mathrm{~K}$ ), ion gun, three ports for evaporators, and LEED-Auger optics. Magneto-optical Kerr effect is measurable in situ with diode laser and variable field coils, for transverse, longitudinal, and perpendicular orientations of magnetization. Four degree of freedom $(x, y, z$, and $\theta)$ manipulator combined with a cryostat allows positioning and cooling (minimum temperature of $150 \mathrm{~K}$ ) of the sample. A room temperature STM chamber is connected to the sample preparation chamber for the real space imaging of the sample surface. ${ }^{13,14}$ Figure 17 presents a typical atomic resolution image acquired at the STM station.

\section{Software features}

Aside from vacuum control and direct access to safety valves and undulators, all the software codes, based on LABVIEW, have been developed in-house to efficiently couple instruments and beamline. The main part is a Windows XP based PC controlling both the monochromators (LE and HE). The movement is based on an intelligent axis control computer board MAXp from Pro-Dex (ex OmsMotion) and the stepper motors are moved via power-motor box (Sincrotrone
Trieste). Positions of monochromators are decoded via Heidenhain optical encoders and readouts via RS-232 interface. Client applications on acquisition computers of both LE and $\mathrm{HE}$ branches talk with the abovementioned server via a TCP protocol on a gigabit dedicated network.

$K$-space tomography acquisition at APE-LE is based on remote control of both the Scienta SES 2002 analyzer and the manipulator. A NI 6602 PCI card controls the steppers motors, while the angular position is read by angular Heidenhain encoder and brought to digital readouts. The acquisition software interfaces directly to lower level Scienta software dynamic link libraries by skipping the main executable (ses.exe) in order to perform automated measurement. Photon energy with the suitable light polarization (monochromator setting and undulator) can be automatically adjusted within the automated measurements. Custom Igor and IDL proce-

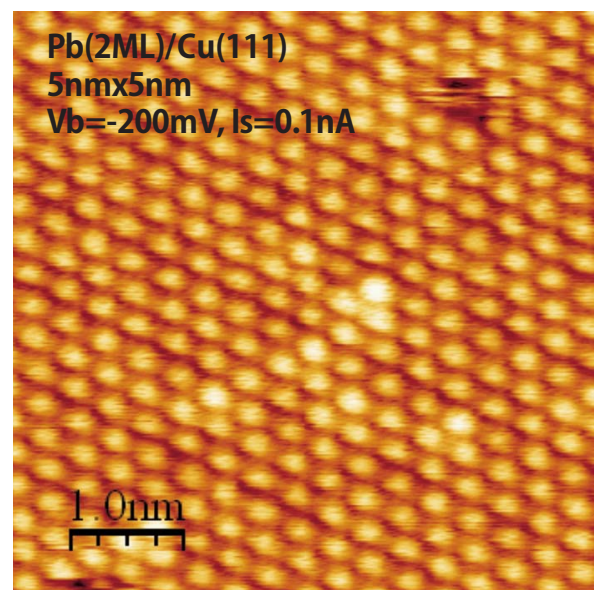

FIG. 17. (Color online) STM topography image with atomic resolution $\left(5 \times 5 \mathrm{~nm}^{2}\right)$. The sample is a $2 \mathrm{ML} \mathrm{Pb}$ thin film epitaxially grown onto a $\mathrm{Cu}(111)$ single crystal $\left(V_{b}=-200 \mathrm{mV}, I_{\text {sample }}=0.1 \mathrm{nA}\right)$. 
dures have been written in order to select the Fermi energy, normalize the intensities levels, and eventually readjust angular mismatch.

APE-HE is also run via custom LABVIEW-based software, with full instrument interfacing (electrometers, power and current supplies, temperature controllers), including Mott-detectors. By taking advantage of ActiveX technology, it is possible to integrate the proprietary electron analyzer software into the LABVIEW projects.

\section{SUMMARY AND CONCLUSIONS}

The APE beamline started its operation on 2003 and was immediately opened to users. Scientists from different fields as chemistry, physics, and materials science have already performed many experiments, and a negligible amount of cross-talk between the two undulator sources has been found during the entire period of operation. The two branches can now be operated independently for roughly $85 \%$ of the available beamtime. Long term users' programs will be therefore possible, corresponding to beamtime access based on usual peer-reviewed procedures but allowing on-line semipermanent growth facilities and access to the off-beam diagnostics.

\section{ACKNOWLEDGMENTS}

We thank all the TASC and Elettra technical services for the enthusiastic and collaborative approach as well as for the engaged participation during all phases of the construction. We acknowledge the fruitful collaboration of the RMP Company and in particular of M. Praitano, F. Lama, and R.
Alessandroni. Thanks are due to those who shared part of the time (and their ideas) with us and in particular to Jörg Kröger, Stefan Egger, Cinzia Cepek, Silvio Modesti, and Andreas Vaterlaus. This work was funded by INFM, progetto Luce di Sincrotrone.

${ }^{1}$ S. Sasaki, Nucl. Instrum. Methods Phys. Res. A 347, 83 (1994).

${ }^{2}$ S. Sasaki, H. Kobayashi, M. Takao, Y. Miyahara, and S. Hashimoto, Rev. Sci. Instrum. 66, 1953 (1995).

${ }^{3}$ B. Diviacco, R. Bracco, D. Millo, R. P. Walker, M. Zalatau, and D. Zangrando, Proceedings of the European Particle Accelerator Conference, Vienna, Austria, 26-30 June 2000 (unpublished).

${ }^{4}$ L. Tosi, B. Diviacco, F. Iazzourene, R. Roux, R. P. Walker, and D. Zangrando, Proceedings of the European Particle Accelerator Conference, Vienna, Austria, 26-30 June 2000 (unpublished).

${ }^{5}$ B. Diviacco, and R. P. Walker, Sincrotrone Trieste Internal Note ST/MTN-97/11.

${ }^{6}$ L. E. Berman, D. P. Siddons, P. A. Montanez, A. Lenhard, and Z. Yin, Rev. Sci. Instrum. 73, 1481 (2002).

${ }^{7}$ D. H. Bilderback, Nucl. Instrum. Methods Phys. Res. A 246, 434 (1986).

${ }^{8}$ D. S. Inosov, R. Schuster, A. A. Kordyuk, J. Fink, S. V. Borisenko, V. B. Zabolotnyy, D. V. Evtushinsky, M. Knupfer, B. Büchner, R. Follath, and H. Berger, Phys. Rev. B 77, 212504 (2008).

${ }^{9} \mathrm{~S}$. Borisenko, 1-cubed ARPES project http://www.ifw-dresden.de/ institutes/iff/research/SC/arpes/1-cubed-arpes.

${ }^{10}$ Swiss Synchrotron Light Source (SLS) (http://sls.web.psi.ch/).

${ }^{11}$ V. N. Petrov, M. Landolt, M. S. Galaktionov, and B. V. Yushenkov, Rev. Sci. Instrum. 68, 4385 (1997).

${ }^{12}$ V. N. Petrov, M. S. Galaktionov, and A. S. Kamochkin, Rev. Sci. Instrum. 72, 3728 (2001).

${ }^{13}$ M. S. Hoogeman, D. Glastra van Loon, R. W. M. Loos, H. G. Ficke, E. de Haas, J. J. van der Linden, H. Zeijlemaker, L. Kuipers, M. F. Chang, M. A. J. Klik, and J. W. M. Frenken, Rev. Sci. Instrum. 69, 2072 (1998).

${ }^{14}$ M. Schmid and P. Varga, Ultramicroscopy 42, 1610 (1992). 